\title{
Efeito residual da cinza de casca de arroz na disponibilidade de silício e fósforo no solo
}

O fósforo $(\mathrm{P})$ é um nutriente limitante para a produção agrícola. Oriundo de fontes não renováveis, estudos que apontem materiais alternativos que forneçam $P$ pode ter grande importância num futuro próximo. Neste sentido, a cinza de casca de arroz surge como uma opção viável devido a sua composição, rica em silício (Si), que pode promover aumento na disponibilidade de $\mathrm{P}$ no solo. $\mathrm{O}$ objetivo deste trabalho foi avaliar o efeito residual da cinza de casca de arroz na disponibilidade de silício e fósforo no solo. Para tal, foi realizado um experimento de campo constituído por dez dosagens de cinza de casca de arroz $0,10,20,30,40,60,80,100,120$ e 140 t ha-1 onde foi realizada uma amostragem de solo aos 15 dias após a aplicação (daa) da cinza, nas camadas de 0,00-0,10 m e 0,10-0,20 m. Para a determinação do efeito residual utilizou-se quatro doses de CCA do experimento inicial referentes a 0, 30, 60 e 120 t ha-1 de onde foram realizadas mais quatro coletas aos 211, 400, 517 e 804 daa. 0 fósforo disponível foi determinado através do método do extrator duplo ácido (Mehlich-1) enquanto que o Si foi determinado pelo método que utiliza o ácido acético como extrator. Os resultados indicaram que a cinza de casca de arroz aumenta as disponibilidades de P e Si no solo; o aumento do Si disponível no solo, extraível pelo ácido acético, equivale a uma fração de no máximo 0,08 \% de Si total da CCA; o efeito residual da CCA na disponibilidade de silício é maior do que na disponibilidade de fósforo; como fonte de fósforo o efeito residual da CCA é menor do que o de fertilizantes minerais solúveis como superfosfatos acidulados e, é possível que a CCA promova a lixiviação de fósforo em solos com baixa capacidade de adsorção do nutriente.

Palavras-chave: Agricultura; Nutrientes; Resíduos.

\section{Residual effect of rice husk ash on availability of silicon and phosphorus in soil}

\begin{abstract}
Phosphorus (P) is a limiting nutrient to agricultural production. Coming from non-renewable sources, studies that point to alternative materials that provide $\mathrm{P}$ may be of great importance in the near future. In this sense, rice husk ash (RHA) can be a viable option due to her composition, rich in silicon (Si), that can increase soil phosphorus availability. This investigation aimed to evaluate the residual effect of rice husk ash in the phosphorus and silicon soil availability. To this end, a field experiment was conducted with ten rice husk ash dosages $0,10,20,30,40,60,80,100,120$ e 140 t ha- 1 where soil sampling was collected at 15 days after ash incorporation, in the layers $0,00-0,10 \mathrm{~m}$ and $0,10-0,20 \mathrm{~m}$. To evaluate residual effect it was used four rice husk ash dosages from initial experiment referring to 0, 30, 60 e 120 t ha- 1 where four more samples are done at 211, 400, 517 and 804 days after ash incorporation. Available phosphorus was determined by the double acid extraction method (Mehlich-1) while silicon was determined by the method that uses acetic acid as extractor. Results showed that rice husk ash increases soil phosphorus and silicon availability; the increased of soil silicon available, extractable by acetic acid, is equivalent to a fraction of a maximum of $0,08 \%$ of total silicon from rice husk ash; the residual effect of RHA in the silicon availability is higher than in the phosphorus availability; as phosphorus source the residual effect of RHA is lower than that of soluble mineral fertilizers such as acidulated superphosphates and, it's possible that RHA causes the leaching of phosphorus in soils with low nutrient adsorption capacity.
\end{abstract}

Keywords: Agriculture; Nutrients; Waste.

\section{Topic: Química Agrícola e Ambiental}

Reviewed anonymously in the process of blind peer.

\section{Aline Hernandez Kath}

Universidade Federal de Pelotas, Brasil

http://lattes.cnpq.br/0321026096496176

alinecou@gmail.com

Juliana Brito da Silva Teixeira

Universidade Federal de Pelotas, Brasil

http://lattes.cnpq.br/6715070315999808

julianabrit@gmail.com

Gláucia Oliveira Islabão

Instituto Federal Farroupilha, Brasi

http://lattes.cnpq.br/3325971276584600

gislabao@gmail.com

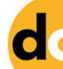

DOI: 10.6008/SPC2179-6858.2017.003.0002
Received: 20/02/2017

Approved: 22/04/2017

Ledemar Carlos Vahl

Universidade Federal de Pelotas, Brasil http://lattes.cnpq.br/8141365816015641 ledovahl@hotmail.com

Referencing this:

KATH, A. H.; TEIXEIRA, J. B. S.; ISLABÃO, G. O.; VAHL, L. C.. Efeito residual da cinza de casca de arroz na disponibilidade de silício e fósforo no solo. Revista Ibero-Americana de Ciências Ambientais, v.8, n.3, p.15-22, 2017. DOI: http://doi.org/10.6008/SPC21796858.2017 .003 .0002 


\section{INTRODUÇÃO}

Os solos brasileiros, de modo geral, têm baixa fertilidade natural em função da elevada acidez, baixa saturação por bases, toxicidade de alguns elementos químicos e baixa disponibilidade de nutrientes. Considerando os nutrientes para o desenvolvimento das plantas, a baixa disponibilidade de fósforo (P) é geralmente considerada a maior limitação (NOVAIS et al., 1999).

A forte afinidade do P com os óxidos e hidróxidos de ferro (goethita e hematita) e de alumínio (gibbsita) do solo tende a reter o P no sistema, tornando-o menos disponível às plantas e à lixiviação. Assim, solos com textura muito argilosa e alto conteúdo de óxidos, apresentam elevada capacidade de adsorção de fosfato, a qual pressupõe ser diferenciada de acordo com as formas dos óxidos de ferro. Entretanto, a magnitude desse fenômeno é influenciada pela natureza e quantidade dos sítios de adsorção, os quais variam de acordo com os fatores intrínsecos e extrínsecos ao próprio solo. Dentre esses fatores, destacam-se a mineralogia, a textura, o pH, o balanço de cargas, a matéria orgânica, o tipo de ácidos orgânicos e a atividade microbiana do solo (BAHIA FILHO et al., 1983).

Estima-se que apenas 5 a 25\% do fósforo solúvel adicionado ao solo, como adubo, seja aproveitado pela cultura que o recebeu e que 75 a 95\% dele seja fixado (ALCARDE et al., 1991). Isso faz com que doses elevadas de fertilizantes fosfatados sejam necessárias para que o teor de fósforo disponível se mantenha em níveis adequados ao desenvolvimento das plantas (BARROW, 1978; GOEDERT et al., 1984).

O fosfato proveniente de rochas fosfáticas tem sido historicamente visto como uma forma abundante e inesgotável de fósforo. Entretanto, estima-se que em meados de 2050 a demanda mundial de fósforo duplique, sendo que alguns estudos apontam que poderá ocorrer um pico na produção de fertilizantes fosfatados nos próximos 30 anos (CORDELL et al., 2011) e um esgotamento das reservas mundiais de rochas fosfáticas nos próximos 50 a 100 anos (CORDELL et al., 1995).

Estudos apontam que a adubação silicatada promove melhorias na disponibilidade do fósforo (P) no solo. Dentre outras variáveis, este fato tem sido atribuído à competição por sítios de adsorção no solo entre os ânions fosfato e silicato (VALLE et al., 2016). O Silício (Si) é o segundo elemento mais abundante da Terra, comumente encontrado na forma de dióxido de silício $\left(\mathrm{SiO}_{2}\right)$ mais conhecido como sílica (KÜHN et al., 2001). O uso de Si na agricultura traz inúmeros benefícios como a redução da toxidade do Fe e Mn (HORST et al., 1978) e o aumento de produtividade em gramíneas (ADATIA et al., 1986).

Diante do exposto - a elevada exigência de fósforo nos solos tropicais, fertilizantes fosfatados serem originados de fontes não renováveis e considerando as interações existentes entre o P e o Si no solo - a cinza de casca de arroz surge como uma possível alternativa eficiente e economicamente viável para suprir estas necessidades.

A cinza de casca de arroz (CCA) - resíduo agroindustrial liberado na queima da casca de arroz - possui composição química média de $423 \mathrm{mg} \mathrm{k}^{-1}$ de silício (Si), além de vários outros elementos em menores concentrações, dentre eles o P (ISLABÃO, 2013). A CCA tem sido amplamente utilizada na agricultura devido a seus comprovados efeitos no aumento do pH (ISLABÃO et al., 2014) e liberação de nutrientes (PAULETTO 
et al., 1990; SANDRINI, 2010). Sendo assim, o objetivo deste trabalho foi avaliar o efeito residual da cinza de casca de arroz na disponibilidade de silício e fósforo.

\section{MATERIAL E MÉTODOS}

O experimento de campo foi conduzido no Centro Agropecuário da Palma, pertencente à Universidade Federal de Pelotas, situado no município do Capão do Leão/RS. O solo do local é um Argissolo Vermelho Amarelo (SEVERO, 1999), de textura franco arenosa, conforme demonstra de forma explícita a tabela 1.

Tabela 1: Atributos químicos e granulometria do solo do experimento nas camadas de 0,00-0,10 m e 0,10-0,20 m.

\begin{tabular}{|c|c|c|c|c|c|c|c|c|c|c|c|}
\hline \multirow[b]{2}{*}{$\mathrm{pH} \mathrm{H} \mathrm{H}_{2} \mathrm{O}$} & \multirow[b]{2}{*}{$\mathrm{CO}$} & \multirow[b]{2}{*}{$\mathrm{P}$} & \multicolumn{4}{|c|}{ Cátions Trocáveis } & \multirow[b]{2}{*}{$\mathrm{CTC}_{7}$} & \multirow[b]{2}{*}{ V } & \multirow{2}{*}{ Argila } & \multirow[b]{2}{*}{ Silte } & \multirow{2}{*}{ Areia } \\
\hline & & & $\mathrm{K}^{+}$ & $\mathrm{Ca}^{+2}$ & $\mathrm{Mg}^{+2}$ & $\mathrm{Na}^{+}$ & & & & & \\
\hline & \multicolumn{2}{|c|}{$\mathrm{g} \mathrm{dm}^{-3} \mathrm{mg} \mathrm{dm}^{-3}$} & \multicolumn{5}{|c|}{\begin{tabular}{l|l}
$\mathrm{Mg}^{+2}$ & $\mathrm{Na}^{+}$ \\
$\mathrm{m} / \mathrm{mol}_{\mathrm{c}} \mathrm{dm}^{-3}$
\end{tabular}} & $\%$ & \multicolumn{3}{|c|}{$\mathrm{g} \mathrm{dm}^{-3}$} \\
\hline$\ldots \ldots$ & \multicolumn{11}{|c|}{. } \\
\hline 5,54 & 11,30 & 5,8 & 1,64 & 23,60 & 10,51 & 0,80 & 76,19 & 49,17 & 169,3 & 224,4 & 606,3 \\
\hline ....... & \multicolumn{11}{|c|}{...0,10-0,20m. } \\
\hline 5,23 & 9,85 & 3,6 & 1,21 & 27,4 & 11,30 & 0,82 & 70,56 & 59,12 & 192,9 & 220,0 & 586,9 \\
\hline
\end{tabular}

Legenda: CO: carbono orgânico; V: saturação por bases. Fonte: Islabão et al. (2014)

O arranjo das unidades experimentais, utilizada na realização do presente trabalho foi a descrita por Islabão et al. (2014). As cinzas provenientes da queima da casca do arroz em leito fluidizado foram fornecidas por uma empresa de Pelotas/RS, de acordo com o ilustrado na tabela 2, que versa sobre a composição química da CCA utilizada no experimento.

Tabela 2: Composição química da CCA utilizada no experimento.

\begin{tabular}{|c|c|c|c|c|c|c|c|c|c|c|c|c|c|c|c|}
\hline $\mathrm{pH}$ & PN & $\mathrm{Si}$ & $\mathrm{K}$ & $\mathrm{Na}$ & $\mathrm{Ca}$ & $\mathrm{Mg}$ & $\mathrm{Al}$ & $C$ & $\mathrm{~N}$ & $\mathrm{P}$ & $S$ & $\mathrm{Cu}$ & $\mathrm{Fe}$ & $\mathrm{Mn}$ & $\mathrm{Zn}$ \\
\hline & $\%$ & \multicolumn{10}{|c|}{$\mathrm{g} \mathrm{kg}^{-1}$} & \multicolumn{4}{|c|}{$\mathrm{mg} \mathrm{kg}^{-1}$} \\
\hline 8,6 & 0,91 & 410,5 & 11,35 & 6,83 & 6,02 & 4,20 & 0,23 & 72,3 & 0,98 & 2,64 & 3,52 & 7,5 & 480 & 1278 & 34 \\
\hline
\end{tabular}

Legenda: PN: poder de neutralização. Fonte: Islabão (2013)

Para a avaliação da disponibilidade de fósforo e silício foram utilizadas as dosagens de CCA equivalentes a 0,10, 20, 30, 40, 60, 80, 100, 120 e $140 \mathrm{t} \mathrm{ha}^{-1}$. A coleta foi realizada aos 15 dias após a aplicação (daa) da CCA. Para o estudo do efeito residual da CCA utilizou-se apenas as doses equivalentes a 0, 30, 60 e $120 \mathrm{t} \mathrm{ha}^{-1}$, conforme tratamento recomendado pela CQFSRS (2004) - tratamento ACR. Essas doses foram selecionadas em função da melhor representatividade dos dados quando distribuídos em gráficos. Para avaliar o efeito do tempo utilizou-se os dados da primeira coleta realizada aos 15 daa e das coletas realizadas aos 211daa, 400daa, 517daa e 804daa.

As coletas foram feitas em duas amostragens independentes em cada profundidade $(0,00-0,10 \mathrm{~m}$ e 0,10-0,20m). Cada amostra foi composta de 6 subamostras coletadas em cada parcela com trado calador. Após, as amostras foram secas em estufa com circulação forçada de ar a $50^{\circ} \mathrm{C}$ até peso constante, destorroadas manualmente com rolo de madeira e passadas em peneira de $2 \mathrm{~mm}$.

As análises químicas do solo foram realizadas nos laboratórios do Departamento de Solos da Universidade Federal de Pelotas. O fósforo disponível foi determinado através do método do extrator duplo 
ácido (Mehlich-1) (TEDESCO et al., 1995). O Si foi determinado pelo método descrito por KORNDÖRFER et al. (2004), que utiliza o ácido acético como extrator.

Os dados foram submetidos à análise de variância pela Two Way ANOVA Repeated Measures que permite avaliar os efeitos das variáveis bem como as interações entre elas. Foram feitas também análises de regressão entre as variáveis e o tempo, cuja significância foi avaliada pelo Teste F. Ambas as análises foram feitas através do programa computacional SigmaPlot. A partir das equações ajustadas através da análise de regressão foram calculados o tempo total $(\mathrm{tt})$ e o tempo de meia vida $\left(t_{0,5}\right)$ dos efeitos dos tratamentos nos atributos químicos.

\section{RESULTADOS E DISCUSSÃO}

\section{Disponibilidade de Si e P}

O aumento na concentração de Si no solo pode ser benéfico para o crescimento e desenvolvimento das plantas, uma vez que são conhecidos os seus benefícios para o aumento da tolerância ao estresse hídrico, aumento da capacidade fotossintética, diminuição do acamamento, redução da transpiração e aumento da resistência ao ataque de pragas e doenças (EPSTEIN, 1994; KORNDÖRFER et al.,1999; KORNDÖRFER et al., 2004).

Apesar deste efeito acentuado da CCA no teor de Si no solo extraível pelo ácido acético, os aumentos verificados nestes teores constituem apenas uma pequena fração do Si total contido na CCA. Considerando que o teor total de Si na CCA utilizada era $410 \mathrm{~g} / \mathrm{kg}^{-1}$, o aumento do Si extraível do solo pelo ácido acético equivaleu a $0,078 \%$ aos 15 daa independente da dose de CCA. Esta fração é da mesma ordem de magnitude da fração do Si da CCA solúvel em água (0,056 \%) constatada por Ueda et al. (2007).

Ao contrário do $\mathrm{Si}$, o aumento do $\mathrm{P}$ extraível promovido pela CCA representou uma fração considerável do $\mathrm{P}$ total contido na CCA e aumentou, linear à dose, de $20 \%$ na dose menor $\left(10 \mathrm{t} / \mathrm{ha}^{-1}\right)$ para 40 $\%$ na dose maior $\left(140 \mathrm{t} \mathrm{ha}^{-1}\right)$. Tal aumento da fração extraível de $\mathrm{P}$ com a dose de CCA pode ser atribuído ao Si da CCA. O Si pode competir com o P pelos sítios de adsorção no solo (OBIHARA et al., 1972) diminuindo a adsorção do $\mathrm{P}$ e, em consequência, aumentando a solubilidade deste nutriente no solo. A similaridade química entre as moléculas de $\mathrm{P}$ e $\mathrm{Si}\left(\mathrm{H}_{2} \mathrm{PO}_{4}{ }^{-}\right.$e $\left.\mathrm{H}_{4} \mathrm{SiO}_{4}{ }^{-}\right)$pode ser a principal forma de explicar a ocorrência deste fenômeno no solo (HINGSTON et al., 1972).

Porém, Islabão et al. (2014) demonstraram os efeitos corretivos da CCA na acidez do solo, logo, assim como Baldeon (1995) afirmou que, no seu experimento, o ganho em P pelas plantas foi devido ao poder alcalinizante do Si e a competição Si x P pelos mesmos sítios de adsorção dos solos, no presente trabalho, o incremento do pH também pode ter promovido o aumento do $\mathrm{P}$ extraível e não apenas o fator competição Si X P isolado. Com o aumento do $\mathrm{pH}$, a carga superficial de partículas do solo torna-se cada vez mais negativa, aumentando a repulsão (menor adsorção) entre fosfato e superfície adsorvente (POSNER et al., 1980), logo, a elevação do pH aumenta a disponibilidade de P.

O teor de silício no solo extraível pelo acetato de amônio aumentou com a dose de CCA aos 15daa na camada superficial de 0,00-0,10m, segundo um modelo de acréscimos decrescentes com as doses 
sucessivas de cinza, tendendo para um valor máximo de $47 \mathrm{mg} / \mathrm{dm}^{-3}$ atingível numa dose $216 \mathrm{t} / \mathrm{ha}^{-1}$ de acordo com a equação quadrática ajustada, conforme se nota na figura 1a. Na camada de 0,10 - 0,20m, houve um pequeno efeito linear da CCA no teor de silício extraível. A pouca movimentação do Si para as camadas mais profundas do solo também foi constatada por Pereira et al. (2003) que utilizaram quatro fontes de Si em experimento conduzido em casa de vegetação e concluíram que as fontes utilizadas aumentaram os teores de Si no solo na camada de $0-0,10 \mathrm{~m}$, sendo que apenas um dos resíduos diferiu estatisticamente da testemunha na camada de 0,10-0,20m.

O teor de P extraível pelo método Mehlich-1 aumentou de forma exponencial com a dose de CCA na camada de 0-0,10 m, como se nota na figura $1 b$. Na camada subjacente de 0,10 - 0,20m, a CCA não afetou o P extraível, o que indica que não houve translocação descendente de $P$, o que era esperado em função da baixa mobilidade deste elemento no solo.

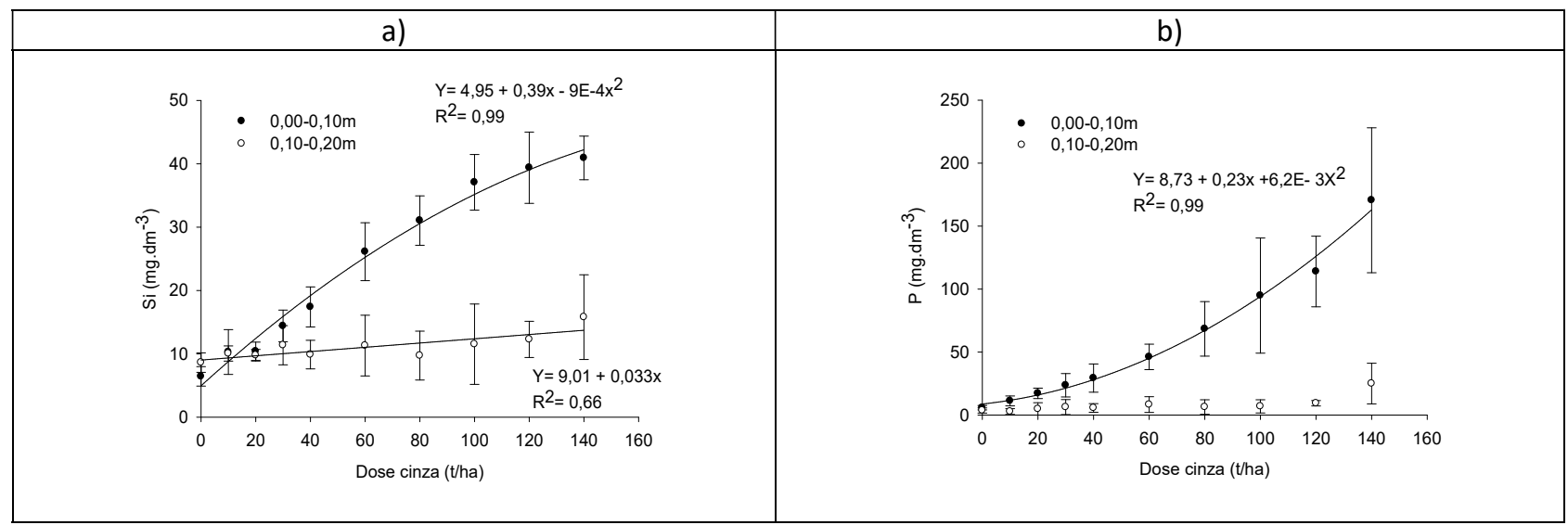

Figura 1. Relação entre doses de cinza de casca de arroz e silício (Si) e fósforo (P) do solo nas camadas de 0,00-0,10 m e 0,10-0,20 m aos 15 dias após a aplicação da CCA.

\section{Efeito residual da CCA}

Para o estudo do efeito residual da CCA, optou-se por utilizar os dados que representam a camada superficial do solo $(0,00-0,20)$ das parcelas com as doses de CCA equivalentes a $0,30,60$ e $120 \mathrm{t} / \mathrm{ha}^{-1}$ e a testemunha ACR. Os efeitos da dose de CCA e do tempo após a sua aplicação nos teores de P e Si foram significativos estatisticamente ao nível menor que $0,1 \%$ de probabilidade, com exceção do efeito da dose no $\mathrm{Si}$, no qual foi significativo a 1,6\%, conforme predisposto na tabela 3 . A interação entre dose e tempo foi significativa para o P em nível menor que 0,1\% e para o Si ao nível de 2,6\%. Isso significa que as variações dos teores de P e de Si com o tempo dependeram da dose de CCA aplicada.

Tabela 3. Níveis de significância obtidos a partir da análise de variância para medidas repetidas (Two - Way Repeated Measures ANOVA) dos elementos $\mathrm{P}$ e $\mathrm{Si}$.

\begin{tabular}{|c|c|c|}
\hline Fator & $\mathrm{P}$ & $\mathrm{Si}$ \\
\hline & \multicolumn{2}{|c|}{$\mathrm{P}$} \\
\hline Dose & $<0,001$ & 0,016 \\
\hline Tempo & $<0,001$ & $<0,001$ \\
\hline Dose x Tempo & $<0,001$ & 0,026 \\
\hline
\end{tabular}

Legenda: P: Probabilidade devido ao acaso.

Mesmo que no Argissolo do experimento, a capacidade de adsorção de P seja baixa, no máximo $230 \mathrm{mg}$ de $\mathrm{P} \mathrm{kg}^{-1}$ de solo (MACHADO, 1993), é suficiente para adsorver grande parte do $\mathrm{P}$ tanto do 
superfosfato simples (tratamento ACR) como do P contido na CCA aplicada. A adsorção pode se tornar mais forte à medida que o tempo passa e isto faz com que o $\mathrm{P}$ extraível diminua com o tempo, como foi observado. Entretanto, na presença da CCA a capacidade de adsorção do P pelo solo pode diminuir devido à sílica contida no material. Segundo Hingston et al. (1972), o ânion $\mathrm{H}_{3} \mathrm{SiO}_{4}^{-}$pode impedir ou dificultar a adsorção de fosfato. Este efeito é mais forte em pH acima de 7,0, mas a adsorção do silicato em pH bem abaixo do $\mathrm{pK}_{\mathrm{a}}$ do $\mathrm{H}_{4} \mathrm{SiO}_{4}$ pode ocorrer devido ao fato de a energia de ligação deste ácido aos óxidos ser suficientemente elevada a ponto de dissociá-lo (OBIHARA et al., 1972) e, portanto, pode diminuir a adsorção do $\mathrm{P}$ mesmo em pH mais baixo do que 7. Embora apenas uma pequena fração do Si da CCA seja solúvel, como o teor deste elemento é muito alto, a quantidade solúvel incorporada ao solo é grande. Isto cria condições para a baixa retenção do P pelo solo, tanto o contido na CCA como o P nativo ou oriundo de fertilizantes. Em função disso, é possível que nos tratamentos com CCA, principalmente nas doses mais altas, a adsorção do P tenha sido muito menor e pelo menos parte da diminuição dos teores de $\mathrm{P}$ com o tempo seja devida à perda de $\mathrm{P}$ por lixiviação, à semelhança dos cátions básicos. A lixiviação do $\mathrm{P}$ não é um fenômeno comum em solos tropicais $\mathrm{e}$ subtropicais devido à alta adsorção.

O P e o Si extraíveis diminuíram exponencialmente com o tempo, exceto na dose zero de CCA, na qual os teores de ambos os elementos foram constantes ao longo do tempo, de acordo com o ilustrado na figura 2. A diminuição dos teores de $\mathrm{P}$, consoante à figura 2a, com o tempo após as adubações, normalmente são consequência da adsorção do ânion fosfato à superfície de óxidos de ferro e alumínio e à formação de fosfatos de $\mathrm{Fe}$, Al ou Ca de baixa solubilidade que se formam no solo. Tanto o $\mathrm{P}$ adsorvido quanto os precipitados de $P$ são sujeitos a um processo de envelhecimento que tornam tais ligações do $P$ mais fortes e menos reversíveis com o tempo.

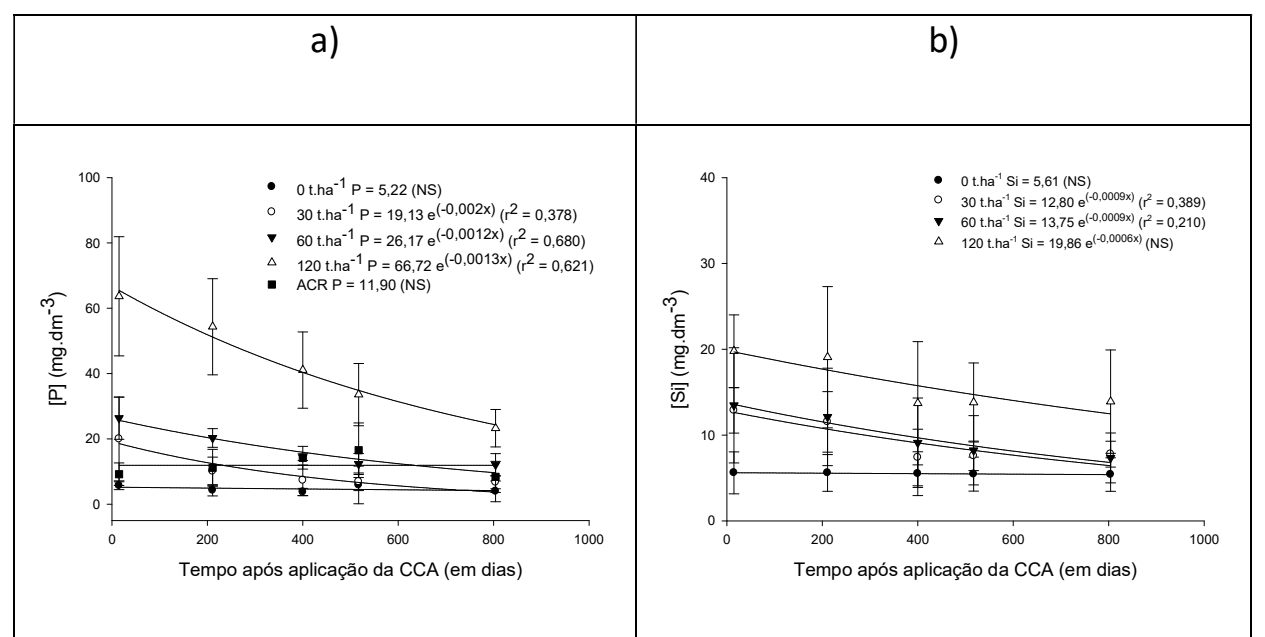

Figura 2: Concentrações de a) P (Mehlich-1) e b) Si (Ácido Acético) em função do tempo após a aplicação de doses de CCA na camada de 0,00-0,20m de um Argissolo.

A diminuição do Si com o tempo, demonstrado na figura $2 b$, embora também tenha seguido um modelo exponencial, nas equações ajustadas as curvaturas foram muito suaves chegando a ser quase lineares. As razões para a diminuição do Si com o tempo podem incluir a formação de polímeros de sílica insolúveis - em pH ácido, o monômero $\left(\mathrm{H}_{4} \mathrm{SiO}_{4}\right)$ polimeriza para formar precipitados de sílica amorfa, o que pode acarretar perda de Si solúvel ocorrendo este fato principalmente quando o teor de Si é alto e o pH baixo 
(ILER, 1979) - e adsorção pois vários compostos comuns no solo são capazes de adsorver Si, entre eles as diversas formas de sílica amorfa, silicatos e substâncias não silicosas como óxidos de Fe, $\mathrm{Al}$ e $\mathrm{Mg}$, os quais apresentam a maior eficiência de adsorção de Si (MCKEAGUE et al., 1963).

Em decorrência disso, o P contido numa dose de CCA de $19,3 \mathrm{t} \mathrm{ha}{ }^{-1}$, que promoveu um aumento inicial do $\mathrm{P}$ extraível equivalente ao aumento promovido pela adubação com superfosfato simples, teve um $\mathrm{t}_{0,5}$ de apenas 168 dias (Tabela 4). É incomparável ao tratamento com superfosfato, no qual o teor de P extraível manteve-se estável durante os 804 dias, sem diminuir.

Tabela 4: Tempo total ( $\mathrm{tt})$ e tempo de meia vida $\left(\mathrm{t}_{0,5}\right)$ estimados para as concentrações de $\mathrm{P}$ e Si retornarem aos valores iniciais do solo.

\begin{tabular}{|c|c|c|c|c|}
\hline Dose & \multicolumn{2}{|c|}{$\mathrm{P}$} & \multicolumn{2}{c|}{$\mathrm{Si}$} \\
\hline & $\mathrm{tt}$ & $\mathrm{t}_{0,5}$ & $\mathrm{tt}$ & $\mathrm{t}_{0,5}$ \\
\hline $\mathrm{tha}^{-1}$ & \multicolumn{4}{|c|}{ Dias } \\
\hline 30 & 649 & 226 & 916 & 366 \\
\hline 60 & 1343 & 426 & 996 & 390 \\
\hline 120 & 1960 & 475 & 1580 & 741 \\
\hline 19,3 & 480 & 168 & - & - \\
\hline
\end{tabular}

\section{CONCLUSÕES}

A cinza de casca de arroz aumenta as disponibilidades de P e Si no solo, avaliado pelos extratores Melich-1 e Ácido acético, respectivamente. Atrelado a isso, tem-se que o aumento do Si disponível no solo, extraível pelo ácido acético, equivale a uma fração de no máximo 0,08 \% de Si total da CCA, independente da dose deste material incorporado ao solo. O efeito residual da CCA na disponibilidade de silício é maior do que na disponibilidade de fósforo. Como fonte de fósforo o efeito residual da CCA é menor do que o de fertilizantes minerais solúveis como superfosfatos acidulados, é possível que a CCA promova a lixiviação de fósforo em solos com baixa capacidade de adsorção do nutriente.

\section{REFERÊNCIAS}

ADATIA, M. H.; BESFORD, A. T.. The effects of silicon on cucumber plants grown in recirculating nutrient solution. Annual Botanical ,v.58, p.343-351, 1986. DOI: https://doi.org/10.1093/oxfordjournals.aob.a087212

ALCARDE, J. C.; GUIDOLIN, J. A.; LOPES, A. S.. Os adubos e a eficiência das adubações. 2 ed. São Paulo: ANDA, 1991.

BAHIA FILHO, A. F. C.; BRAGA, J. M.; RESENDE, M.; RIBEIRO, A. C.. Relação entre adsorção de fósforo e componentes mineralógicos da fração argila de Latossolos do Planalto Central. Revista Brasileira de Ciências do Solo, v.7, p.221226, 1983.

BALDEON, J. R. M.. Efeito da ação alcalinizante e da competição entre silicato e fosfato na eficiência do termofosfato magnesiano em solos ácidos. Tese (Doutorado em Ciências do Solo) - Universidade de São Paulo, Piracicaba, 1995.

BARROW, N. J.. The description of phosphorus adsorption curves. Soil Science, v.29, p.447- 462, 1978.

DOI: http://10.1111/j.1365-2389.1978.tb00794.x
CHANDRASEKHAR, S.; PRAMADA, P. N.; PRAVEEN, L.. Effect of calcination temperature and heating rate on the optical properties and reactivity of rice husk ash. Journal of Materials Science, v.40, p.6535-44, 2005. DOI: http://10.1007/s10853-005-1816-z

CORDELL, D.; DRANGERT, J. O.; WHITE, S.. The story of phosphorus: global food security and food for thought. Global Environmental Change, Oxford, v.19, n.2, p.292-305, 2009. DOI: http://10.1016/j.gloenvcha.2008.10.009

CORDELL, D.; WHITE, S.. Peak Phosphorus: clarifying the key issues of a vigorous debate about long-term phosphorus security. Sustainability. v.3, p.2027-2049, 2011. DOI: http://10.3390/su31020227

CQFSRS. Comissão de Química e Fertilidade do Solo. Manual de adubação e calagem para os Estados do Rio Grande do Sul e Santa Catarina. Porto Alegre: Sociedade Brasileira de Ciência do Solo, 2004.

EPSTEIN, E.. The anomaly of silicon in plant biology. Proceedings of the National Academy of Science of the United States of America, v.91, p.11-17, 1994. DOI: http://10.1073/pnas.91.1.11 
GOEDERT, W. J.; LOBATO, E.. Avaliação agronômica de fosfatos naturais em solo de Cerrado. Revista Brasileira de Ciências do Solo, v.8, p.97-102, 1984.

HINGSTON, F. J.; POSNER, A. M.; QUIRK, J. P.. Anion adsorption by goethite and gibsite. I. The role of the proton in determining adsorption envelopes. Journal Soil Science, v.23; p.177-192, 1972. DOI: http://10.1111/j.13652389.1972.tb01652.x

HORST, W. J.; MARSCHNER, H.. Symptome von ManganÜberschuß bei Bohnen (Phaseolus vulgaris L.). Journal of Plant Nutrition and Soil Science, v.141, p.129-142, 1978. DOI: http://10.1002/ipln.19781410202

ILER, R. K..The chemistry of silica: Solubility, Polymerization, Colloid and Surface Properties and Biochemistry of Silica. 1 ed. New York: Wiley-Interscience, 1979.

ISLABÃO, G. O.. Uso da cinza de casca de arroz como corretivo e condicionador do solo. Tese (Doutorado em Ciências) - Universidade Federal de Pelotas, Pelotas, 2013.

ISLABÃO, G. O.; VAHL, L. C.; TIMM, L. C.; PAUL, D. L.; KATH, A. H.. Rice husk ash as corrective of soil acidity. Revista Brasileira de Ciências do Solo, v.41, p.934-941, 2014. DOI: http://doi.org.10.1590/S0100-06832014000300025

KORNDÖRFER, G. H.. Fósforo na cultura da cana de açúcar. In: YAMADA, T.; ABDALLA, S. R. S.. In: SIMPÓSIO SOBRE FÓSFORO NA AGRICULTURA BRASILEIRA. Anais. Piracicaba: Associação Brasileira para Pesquisa da Potassa e do Fosfato, 2004.

KORNDÖRFER, G. H.; ARANTES, V. A.; CORRÊA, G. F.; SNYDER, G. H.. Efeito do silicato de cálcio no teor de silício no solo e na produção de grãos de arroz de sequeiro. Revista Brasileira de Ciências do Solo, v.23, p.635-641, 1999. DOI: http://doi.org/10.1590/S0100-06831999000300017

KORNDÖRFER, G. H.; PEREIRA, H. S.; NOLLA, A.. Análise de silício: solo, planta e fertilizante. Uberlândia: Universidade Federal de Uberlândia, 2004.

KÜHN, I. D.; HOTZA, D.. Caracterização de cinza de casca de arroz para uso como matéria-prima na fabricação de refratários de sílica. Química Nova, v.24, p.778-782, 2001. DOI: http://dx.doi.org/10.1590/S0100-40422001000600013

MACHADO, M. I. C. S.; BRAUNER, J. L.; VIANNA, A. C. T.. Formas de fósforo na camada arável de solos do Rio Grande do Sul. Revista Brasileira de Ciência do Solo, Campinas, v.17, p.331-336, 1993.

MCKEAUGUE, J.; CLINE, M. G.. Silica in soil solutions. II The adsorption of monosilic acid by soil and by other substances.
Canadian Journal of Soil Science, v.43. p.83 -96, 1963. DOI: http://10.4141/cjss63-01

NOVAIS, R. F.; SMYTH, T. J.. Fósforo em solo e planta em condições tropicais. 1 ed. Viçosa: Universidade Federal de Viçosa, 1999.

OBIHARA, C. H.; RUSSEL, E. W.. Specific adsorption of silicate and phosphate by soils. European Journal of Soil Science, v.23, n.1, p.105-117, 1972. DOI: http://10.1111/j.13652389.1972.tb01646.x

PAULETTO, E. A.; NACHTIGALL, G. R.; GUADAGNIN, C. A.. Adição de cinza de casca de arroz em dois solos do município de Pelotas, RS. Revista Brasileira de Ciência do Solo, v.14, p.255-258, 1990.

PEREIRA, H. S.; VITTI, G. C.; KORNDÖRFER, G. H..

Comportamento de diferentes fontes de silício no solo e na cultura do tomateiro. Revista Brasileira de Ciências do Solo, v.27, p.101-108, 2003. DOI:

http://dx.doi.org/10.1590/S0100-06832003000100011

POSNER, A. M.; BOWDEN, J. W.. Adsorption isotherms: should they be split?. Soil Science Society of America Journal, Madison, v.31, p.1-10, 1980. DOI: http://10.1111/j.1365-2389.1980.tb02059.x

SANDRINI, W. C.. Alterações químicas e microbiológicas do solo decorrentes da adição de cinza de casca de arroz. Dissertação (Mestrado em Ciências) - Universidade Federal de Pelotas, Pelotas, 2010.

SEVERO, C. R. S.. Caracterização dos solos do Centro Agropecuário da Palma. Dissertação (Mestrado em Agronomia) - Universidade Federal de Pelotas, Pelotas, 1999.

TEDESCO, M. J.; GIANELLO, C.; BIASSANI, C. A.; BOHNEN, H.; VOLKWEISS, S. J.. Análises de solo, plantas e outros materiais. Porto Alegre: Universidade Federal do Rio Grande do Sul, 1995.

UEDA, T.; KUNIMITSU, Y.; SHINOGI, Y.. Potential conflicts for the reuse of rice husk in Thailand. Paddy and Water Environment, v.5, p.123-129, 2007. DOI: http://10.1007/s10333-007-0069-7

VALLE, L. A. R.; RODRIGUES, S. L.; RAMOS, S. J.; PEREIRA, H. S.; AMARAL D. C.; SIQUEIRA, J. O.; GUILHERME, L. R. G.. Beneficial use of a by-product from the phosphate fertilizer industry in tropical soils: effects on soil properties and maize and soybean growth. Journal of Cleaner Production, Oxford, v.112, p.113-120, 2016. DOI:

http://doi.org/10.1016/j.jclepro.2015.07.037 